\title{
Identification of Stock Market Manipulation with Deep Learning
}

This paper was downloaded from TechRxiv (https://www.techrxiv.org).

\section{LICENSE}

CC BY 4.0

SUBMISSION DATE / POSTED DATE

03-02-2022 / 07-02-2022

\section{CITATION}

Tallboys, Jillian; ZHU, YE; Rajasegarar, Sutharshan (2022): Identification of Stock Market Manipulation with Deep Learning. TechRxiv. Preprint. https://doi.org/10.36227/techrxiv.19111730.v1

$\mathrm{DOI}$ 


\title{
Identification of Stock Market Manipulation with Deep Learning
}

\author{
Jillian Tallboys $(\bowtie)$, Ye Zhu, and Sutharshan Rajasegarar \\ School of Information Technology \\ Deakin University, Geelong, Victoria, Australia \\ tallboysjillian@gmail.com
}

\begin{abstract}
Anomaly detection is a common and critical data mining task, it seeks to identify observations that differ significantly from others. Anomalies may indicate rare but significant events that require action. Market manipulation is an activity that undermines stock markets worldwide. This paper shares five large real-world, labelled data sets of anomalous stock market data where market manipulation is alleged to have occurred. Cutting edge deep learning techniques are then shown to successfully detect the anomalous periods. An LSTM based method with dynamic thresholding is particularly promising in this domain as it was able to identify contextual local anomalies in the data quickly, taking seconds to score two years of trading data for each stock, which can often be a challenge for deep learning approaches.
\end{abstract}

Keywords: Anomaly Detection · Time Series · Deep Learning · Stock Market.

\section{Introduction}

Anomaly detection, seeks to identify observations that differ significantly from others in a data set. An anomaly is best defined as "an observation that deviates so significantly from other observations as to arouse suspicion that it was generated by a different mechanism" [10]. Depending on the domain, these anomalies can represent adverse actions like fraud, mechanical failure [21] or illness [4].

In time series data there are broadly three kinds of anomalies: Point anomaly, where a single observation has an unusual value [7]; Collective or segment anomaly, where a number of observations are considered anomalous as a group but do not individually have usual values [5]; Contextual or Local anomaly: observations are only anomalous relative to neighbouring data points but not an anomaly relative to all other observations [12].

Identifying anomalies in a time series is a significant challenge, as the data has the inherently complex characteristics of seasonality, periodicity, trend and irregularity [10]. This problem is further complicated by:

- the lack of labelled data suitable for training supervised methods, which even when available, can be impractical for large data sets with high frequency observations [12] 
- even if labelled, by their nature anomalies are rare and this gives rise to a highly unbalanced classes in the data set [12]

Deep learning approaches are a subset of Machine Learning techniques that are based on artificial neural network architectures. These approaches have been used for both regression and classification problems across many domains such as computer vision, speech recognition and language processing. Their broad application is due to their ability to automatically discover complex features without extensive domain knowledge [11]. Given time series' data complex characteristics, it is no surprise these techniques are being explored to detect anomalies.

Although there has been extensive prior work on anomaly detection, approaches for time series data are less often considered. However, there has been a growing body of work that considers anomaly detection for time series data using deep learning approaches across many domains. Identifying anomalies in stock markets could benefit from such approaches, as anomalies periods that may be associated with market manipulation [6]. With around 33 million trades per month on the Australian Stock Exchange [1], effective and efficient identification of activities that may indicate market manipulation is necessary.

This paper will focus on techniques for unsupervised time series anomaly detection. Unsupervised approaches assume no a priori knowledge of anomalies, no normal baselines for comparison and no clear segmentation possible (e.g. no strong periodic patterns say for example as in an electrocardiogram signal) [5]. This most accurately represents real-life scenarios where it is impractical to label large volumes of streamed data (including stock market price data) [11]. However, to enable comparison between methodologies, the data sets used in such papers contain known anomalies. These are identified by human expert or synthetically added by algorithm.

Further, an efficient anomaly detection approach needs to be able to:

1. Detect anomalies quickly, that is it does not take long to train or score the observations, which can be a challenge with deep learning approaches.

2. Detect different types of anomalies, at a minimum it should be able to identify point and contextual anomalies as these are most common in this use case.

In the next section, this paper will outline traditional and cutting-edge approaches. Then five large real-world, labelled data sets of anomalous stock market data will be shared and discussed, contributing to the field as such data is rarely publicly available. Next, this paper demonstrates that market manipulation is able to be detected using two cutting-edge deep learning approaches, TadGAN and LSTM with Dynamic Thresholding, and with results compared with a more traditional ARIMA approach. This paper will then evaluate the results and suggest improvements and identify any drawbacks in using the candidate methods.

\section{Related Work}

Artificial Neural Networks have been used since 1988 to predict stock returns and have shown to be advantageous as they are numeric, data-driven and adap- 
tive with a higher capacity for noisy, nonlinear data [22]. With deep learning these advantages have been enhanced as deep nonlinear topologies can be built to better fit highly nonlinear functions [22]. Anomalies may indicate market manipulation, which is of great interest to markets and regulators. However, there has not been much research on applying deep learning approaches to identifying anomalies in stock markets. This section contains a summary of the small number of papers found from the last five years.

In Islam et al. [8], their ANOMALOUS algorithm is trained on historical stock volume data of US companies that had been identified from litigation as prominent illegal insider trading cases. In their ANOMALOUS algorithm, they used a LSTM (long short-term memory) RNN (recurrent neural network) to predict a future window of transaction volume. To identify the anomalies, Normalised Cross Correlation was used to measure the similarity of the signals. Unfortunately comparisons to other approaches were not shared.

Wang et al.[20] propose a RNN-based ensemble learning (RNN-EL) framework. This takes a multi-dimensional approach and combines features from trading records and company characteristics from the China Securities Regulatory Commission, which have been labelled using prosecuted cases. Their results show a results show the method outperforms the 8 comparison machine learning and statistical approaches by $29.8 \%$ in terms of AUC value.

In contrast to the above supervised approaches, Leangarun et al. [9] have developed an unsupervised approach using LSTM-GANs (generative adversarial networks). Based on LSTM to learn normal behaviour, the discriminator network of GANs detects the anomalies. They synthesised and injected anomalous observations into the data. It achieved $68.1 \%$ accuracy on unseen market data, identifying "pump-and-dump" manipulations. This approach seems quite promising as it takes the LSTM architecture as a base, which seems to be the best choice for time series data.

From the works above, it appears that deep learning techniques tend to be superior to more traditional statistical and machine learning techniques for identifying anomalies in time series. This is shown particularly in Munir's et al. work [10], comparing a wide range of techniques. In particular LSTM and GAN architectures seem to be particularly successful, given this TadGAN and LSTM with Dynamic Thresholding have been tested with stock market data in section 3.4 .

While identifying market manipulation is a concern to markets and regulators, it appears most of the work using deep learning approaches on stock market data focuses on forecasting and prediction rather than identifying anomalies. There is a gap here in the research, as only a small number of papers identifying market manipulation apply deep learning techniques, and they mostly rely on supervised techniques with labelled data. Labelled data is difficult and costly to produce, and may limit the model's application in that it is only trained on identified cases of known manipulation. In the next section we identify five real-world cases of alleged market manipulation to be used to test the selected approaches. 


\section{Stock market manipulation identification}

This paper will determine if deep learning techniques can be utilised to identify anomalies that may signify market manipulation in stock market trading data. It will compare two promising techniques for unsupervised learning - TadGAN and LSTM with Dynamic Thresholding with a statistical approach, ARIMA. These will be tested on stock market data with real market manipulation anomalies labelled for comparison purposes. The algorithms will also be assessed on their efficiency, their speed and the breadth of anomaly types identified, as well as their accuracy. This paper will identify any drawbacks in using these approaches to identify stock market manipulation. The approach taken is summarised in figure 1.

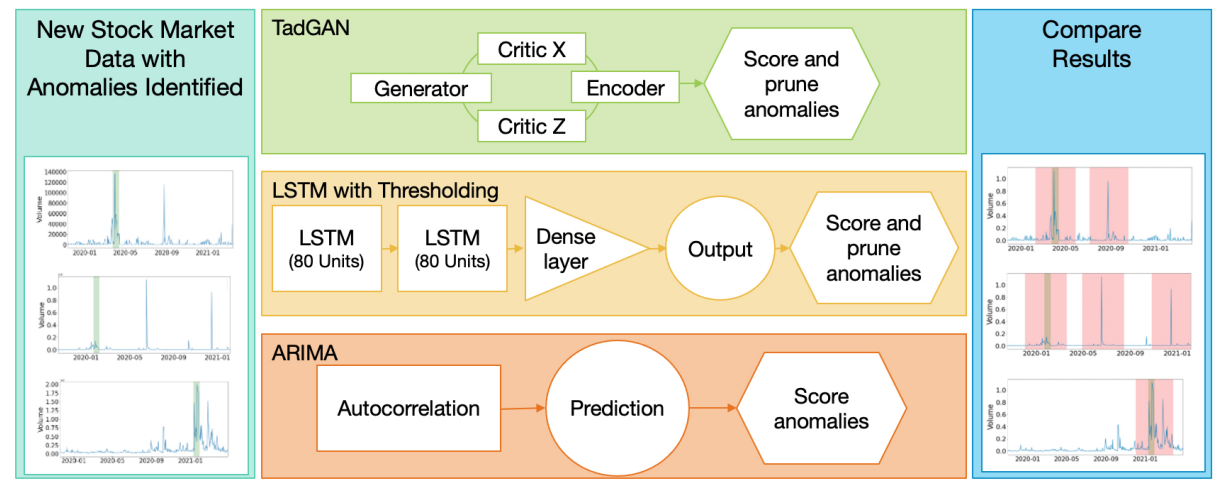

Fig. 1. Five new data sets were sourced and known anomalies were identified, these were then fed to the selected approaches and results were compared.

\subsection{New Stock Market Data for Anomaly Detection}

Stock market regulators such as the Australian Investment and Securities Commission (ASIC) and the Securities \& Exchange Commission in the US seek to ensure the markets they regulate are fair, strong and efficient [2]. This includes administering and enforcing the law against misconduct. Both ASIC and the SEC publicly share details of recent cases on their official websites, asic.gov.au and sec.gov. These were searched with phrases such as "market manipulation" as well as "pump and dump". A "pump and dump" occurs when stocks are quickly sold while a stock's price is high based on false or misleading statements [8].

Unfortunately there were only a few results available on ASIC and insufficient information was publicly available on the exact dates and impacts of the stock manipulation. The SEC, however provide significant detail including copies of the court filings. These documents include dates of the suspicious trades or inaccurate announcements. The SEC has also released a bulletin, warning investors 
of COVID-19 related "pump and dump" scams, and included a list of stocks suspended from trade on suspicion of releasing information was inaccurate or unreliable [19]. From this, four recent cases were selected, including two where charges were laid.

In addition, in early 2021 there was much media attention paid to a social media lead "short squeeze" (a type of pump and dump manipulation) on the US retailer, GameStop. This as also been included for experimentation.

A summary of each stock is provided in table 1 and an chart of each time series with the market manipulation noted is shown at Figure 2.

Turbo Global Partners, Inc. ("TRBO") This case involves two COVID19 related fraudulent press releases issued on 30 March and 3 April 2020 by TRBO [17]. These misleading releases materially affected the trading market for TRBO stock, where trading volume doubled and the intraday high for the share price jumped by around $15 \%{ }^{1}$. As a result the SEC suspended trade on 9 April 2020, which we will assume marks the end of anomalous period ${ }^{2}$.

Applied Biosciences Corp ("APPB") In press releases on 25 and 31 March 2020, APPB made fraudulent claims that it was offering and shipping COVID-19 related products including a finger-prick COVID-19 test to the general public [18]. These statements materially affected trade, the stock price increased from $\$ 0.45$ to $\$ 0.80$, and its volume increased from 1,600 to 136,300 shares sold, between 31 March 2020 to 1 April $2020^{3}$. As a result the SEC suspended trade on 13 April 2020, which we will assume marks the end of anomalous period $^{4}$.

Aethlon Medical, Inc. ("AEMD") On 7 February 2020 trade was suspend for AEMD. This was due to potentially inaccurate information relating to the viability of the company's product to treat the coronavirus and unusual market activity by third-party promoters since 22 January 2020. No charges to date have been laid ${ }^{5}$.

No Borders, Inc. ("NBDR") From 11 March 2020, NBDR made potentially misleading social media statements and press releases about it's COVID-19 specimen collection kits and personal protective equipment products being available for distribution in the United States. The SEC suspended trade on 3 April 2020. No charges to date have been laid ${ }^{6}$.

GameStop ("GME") Users from the online community r/wallstreetbets on Reddit.com executed a short squeeze on GME in January 2021. These actions rapidly increased GME's stock price and caught the attention of the SEC, the US government and the media. On 11 January 2021, GME appointed three new directors to its board which was well received by the market. By 13 January 2021 , the stock price had risen by $50 \%$ to $\$ 31.40$ a share and this generated

\footnotetext{
${ }^{1}$ https://www.sec.gov/litigation/complaints/2020/comp-pr2020-111-turbo.pdf

2 https://www.sec.gov/litigation/suspensions/2020/34-88609-o.pdf

3 https://www.sec.gov/litigation/complaints/2020/comp24977.pdf

${ }^{4}$ https://www.sec.gov/litigation/suspensions/2020/34-88627-o.pdf

${ }^{5}$ https://www.sec.gov/litigation/suspensions/2020/34-88142-o.pdf

${ }^{6}$ https://www.sec.gov/litigation/suspensions/2020/34-88549-o.pdf
} 
interest on r/wallstreetbets, particularly posts by an individual investor, Keith Gill, who had previously shared his analysis of the stock as undervalued. On 19 January 2021, Citron Research (a stock research firm) called GME buyers 'suckers' on Twitter, momentum grew for GME on r/wallstreetbets driving the price up another $50 \%$. The momentum in GME stocks continued, with prices reaching $\$ 354.83$ after a tweet by Elon Musk on 26 January 2021 [16].

The rising price put some hedge funds in a precarious position. Citron Capital and Melvin Capital, two firms who shorted GME, closed their positions on 27 January 2021 taking significant losses. As a result, a number of trading platforms restricted trades involving GME. By 29 January 2021, lawmakers and the SEC publicly made statements and the price fell to $\$ 112.25$. The price remained volatile but trended down to $\$ 53.33$ a share by 4 February 2021 [16].

While the SEC has not categorised the influence of Reddit users as market manipulation [16], the short squeeze on GME is an interesting anomaly for investigation. For this research we will focus on the time period between $11-29$ January 2021.

\subsection{Source}

These five stocks were used to test if such anomalies are detectable and compare the efficacy of the approaches. Data for these stocks were retrieved via the yfinance library in Python. This library uses the Yahoo! Finance API and returns daily stock data (open, high, low, close and volume) for the selected period. For this research, data for 24 months before the identified market manipulation was selected, and data to 12 months after where available (N.B. given the recency of GME's anomalies, only three months of data after the anomaly was available).

\subsection{Anomalies}

Real anomalies were manually flagged as followed based on court filings and media reporting as summarised in Table 1.

Table 1. Real Anomaly Dates

\begin{tabular}{|l|l|l|l|l|l|}
\hline Stock & TRBO & APPB & AEMD & NBDR & GME \\
\hline Anomaly Start & 30-Mar-20 & 25-Mar-20 & 22-Jan-20 & 11-Mar-20 & 11-Jan-21 \\
\hline Anomaly End & 9-Apr-20 & 13-Apr-20 & 7-Feb-20 & 3-Apr-20 & 29-Jan-21 \\
\hline
\end{tabular}

It is to be expected that other anomalies will be present in the data, representing normal market responses to the company's behaviour (e.g. changes in leadership, profit announcements). In a practical application of the approach, the detected anomalies should be cross checked with a service that provides a feed of such announcements (e.g. Bloomberg). 


\subsection{Approaches applied}

The following approaches were applied to each data set.

TadGAN The approach described in Geiger et al. [5], uses a Generative Adversarial Network (GAN). GANs are often used in image recognition, where a neural network is used to generate a fake image with random noise (the Generator) to fool another neural network who distinguishes between the real a fake images (the Critic). In their paper, this process is leveraged for time series where the generator trains on the observed data and is used to reconstruct the time series. The z-scores of both the error between the real and reconstructed time series as well as the Critic outputs are used to score anomalies. A threshold of 4 standard deviations from the mean initially identifies anomaly windows, which are then pruned to minimise false positives. The code for this approach is available in a Python library called Orion ${ }^{7}$. A summary of the underlying GAN architecture used in the TadGAN approach is shown in figure 1.

By default TadGAN takes inputs with sequences of length 100, the latent space is 20-dimensional and the batch size is 64 . It uses a 1-layer bidirectional LSTM with 100 hidden units as Encoder, while the Generator uses a 2-layer bidirectional LSTM with 64 hidden units. A 1-D convolutional layer is used for Critics [5]. Epochs, the number of times the full data set is cycled through the model, were trialed at 35 and 70 . The window size, sets the size of the subsegments, was set at the default of 100 as this parameter is not tunable in the current Orion library implementation.

LSTM with Dynamic Thresholding The approach described by Hundman et al. [7], uses an LSTM to make predictions and a nonparametric, unsupervised approach to find an error threshold to identify both point and contextual anomalies. LSTMs contain a weighted self-loop that enables them to consider both the recent and more long-term dependencies in the data. This makes them a robust approach to highly complex temporal data. The threshold that is found in the approach considers the smoothed errors from the LSTM prediction, and includes a pruning step that ensures only the anomalies that cause the greatest change in the mean and standard deviation of the smoothed errors are flagged. The code for this approach has also been implemented in the Orion library.

By default the LSTM with Dynamic Thresholding takes inputs with sequences of length 250 and a batch size of 64 . The architecture includes two LSTM layers with 80 hidden units each, then a dense layer with one unit which is used to predict the next time step value [7].

A summary of this approach is shown in Figure 1. Epochs were trialed at 35 and 70 . The window size was tested at 10 and 250 .

ARIMA Autoregressive Integrated Moving Average (ARIMA) is a statistical technique commonly used in time series analysis and anomaly detection [3]. This makes it a useful benchmark for comparison of the two methods above. It predicts future values from autocorrelations, and uses point-wise prediction errors that exceed the confidence band to detect anomalies [5]. The implemen-

\footnotetext{
$\overline{7}$ https://github.com/signals-dev/Orion
} 
tation from the Orion library has been applied. The window size was tested at 10,100 and 250 .

\subsection{Environment}

The approaches were applied in Google Colaboratory, a hosted Jupyter notebook service with 2 Intel(R) Xeon(R) CPU @ 2.30GHz and 13GB of RAM. The Adam optimiser was used for all approaches.

\section{Results \& Discussion}

In both Geiger et al. [5] and Hundman et al. [7], the following approach is used to the calculation of evaluation metrics:

- True positive (TP) if the real anomaly overlaps with any detected anomaly window

- False negative (FN) if the real anomaly does not overlap with any detected anomaly window

- True negative (TN) if the real anomaly overlaps with any detected anomaly window

- False positive (FP) if detected anomaly window does not overlap with any real anomaly

While this approach does penalise false positive windows, it is somewhat lenient as a true positive is granted if the real anomaly falls anywhere in the detected window. In the Orion library a stricter, weighted approach to evaluation is available. In this approach each window is considered in a window to window comparison and then weighted by duration [5]. This penalises the approach when the detected window is larger (or smaller) than the real anomaly. Both the overlapping and weighted metrics described above have been included in the evaluation in the calculation of the following metrics: $F 1=\frac{2 \cdot \text { Precision } \cdot \text { Recall }}{\text { Precision }+ \text { Recall }}$, where Precision $=\frac{T P}{T P+F P}$ and Recall $=\frac{T P}{T P+F N}$.

In most cases, all approaches were able to correctly identify the anomalous area. However, the ARIMA approaches tended to provide a smaller detection window and so had a higher weighted F1, Precision and Recall scores than the LSTM with Dynamic Thresholding or TadGAN approaches.

The best approaches and their respective parameter settings for each data set are shown in Table 2, while Figure 2 visualises this output.

From the results it appears deep learning approaches work well to identify anomalies in stock market data. However, a more traditional statistical approach, ARIMA out performs the deep learning approaches in terms of speed and more specifically identifying the anomalous period. That said, ARIMA did not perform well identifying the contextual / local anomaly in the AEMD data set.

For the AEMD data set the best approach was LSTM with Dynamic Thresholding. This approach shows real promise in that it was successfully able to detect the real anomaly being of the contextual / local anomaly type (see figure 2). In 
Table 2. Best approach for each data set

\begin{tabular}{|l|l|c|c|}
\hline Stock & Best approach & F1-score & Elapsed time \\
\hline TRBO & ARIMA, Window Size $=250$ & 0.14 & $24.7 \mathrm{~s}$ \\
\hline APPB & ARIMA, Window Size $=250$ & 0.16 & $24.5 \mathrm{~s}$ \\
\hline AEMD & LSTM, Epochs $=35$, Window Size $=10$ & 0.09 & $19 \mathrm{~s}$ \\
\hline NBDR & ARIMA, Window Size $=250$ & 0.33 & $23.7 \mathrm{~s}$ \\
\hline GME & ARIMA, Window Size $=100$ & 0.27 & $27 \mathrm{~s}$ \\
\hline
\end{tabular}

this data set the volumes during the period of alleged market manipulation are not as extreme as two other periods in the time series, which relate to positive company announcements regarding FDA approvals [13] and clinical trials [14]. This is an important feature as stocks can be quite volatile and responsive to forces that are not market manipulation such as announcements that may affect future profits (as in this case).

In terms of speed, ARIMA over all was the fastest approach to apply with the elased time of less than a minute for each run, followed by LSTM with Thresholding with no more than 3 minutes 9 seconds elasped while TadGAN took up to 15 minutes 20 seconds depending on the number of epochs and the data set used.

It should be noted that while the detected segments appear large and the weighed scores are low, identifying market manipulation is like searching for a needle in a haystack given the high volumes of stocks and data. Currently, regulators such as the SEC and ASIC rely on tip-offs from the public which are then investigated [15]. In a practical application, this type of model would feed into a detection system and be collated with other data sources for human investigation. For example if an anomalous period coincided with a merger announcement (which would be available from a regulatory system) it could be disregarded by investigators. Further, the anomaly dates specified only relate to actions that can be proven but the market manipulation may have started earlier.

For the purposes of identifying market manipulation using both ARIMA and LSTM with dynamic thresholds would be prudent. The idea of combining both deep learning and statistical techniques is outlined in Buda et al. [3] approach, DeepAD, which combines LSTM, ARIMA and Holt-Winters models to identify anomalies, and may perform better than the approaches selected on these data sets.

Limitations The TadGAN approach described in Geiger et al. [5], was applied using the Orion library. Unfortunately, the window size parameter was unable to be adjusted, and remained at the default 250 observations. Developing new code to remedy this was unfeasible in the time frame for this research project. While adjusting this parameter may have resulted in higher evaluation scores, approach selection would unlikely change as ARIMA and LSTM with Dynamic Thresholding generally outperformed TadGAN. 
Traded volumes for Turbo Global Partners, Inc. (“TRBO”)

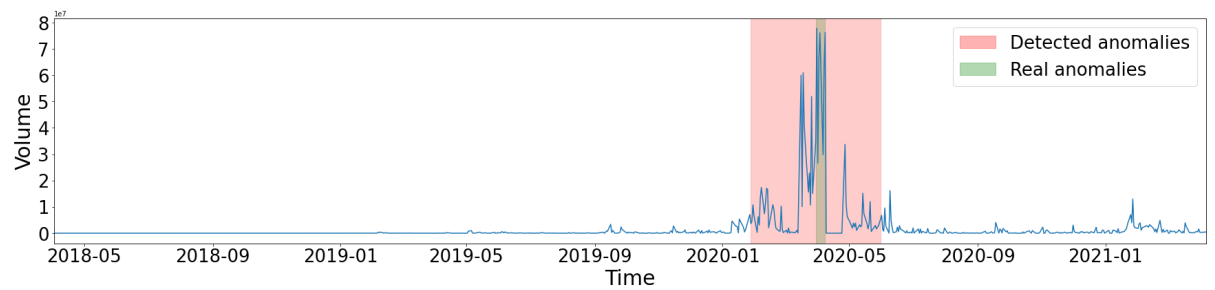

Traded volumes for Applied Biosciences Corp (“APPB”)

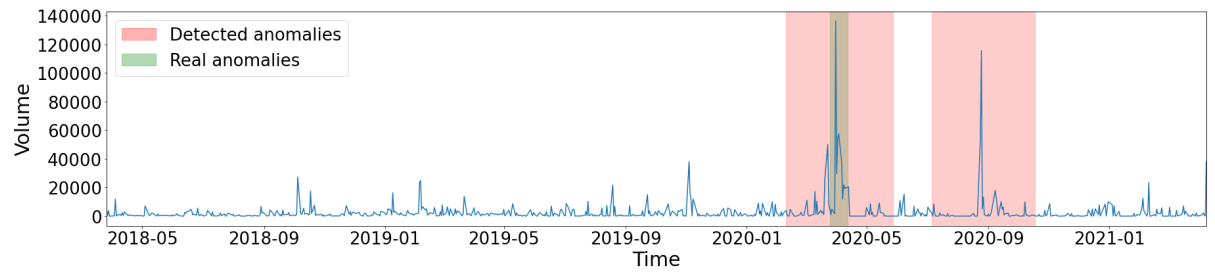

Traded volumes for Aethlon Medical, Inc. (“AEMD”)

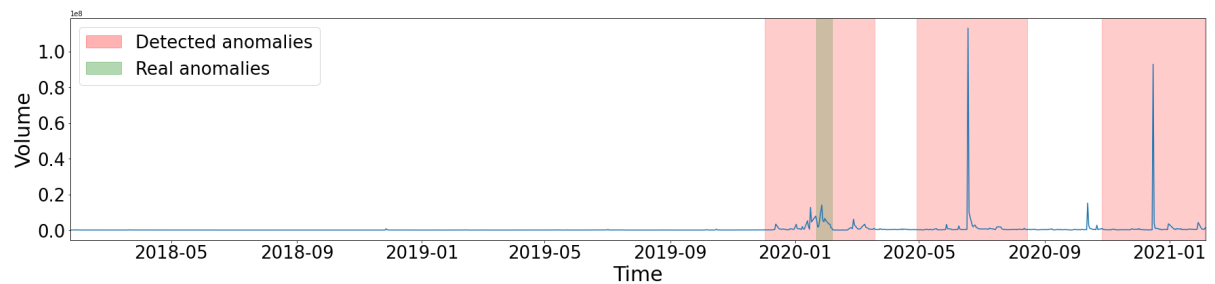

Traded volumes for No Borders, Inc. (“NBDR”)

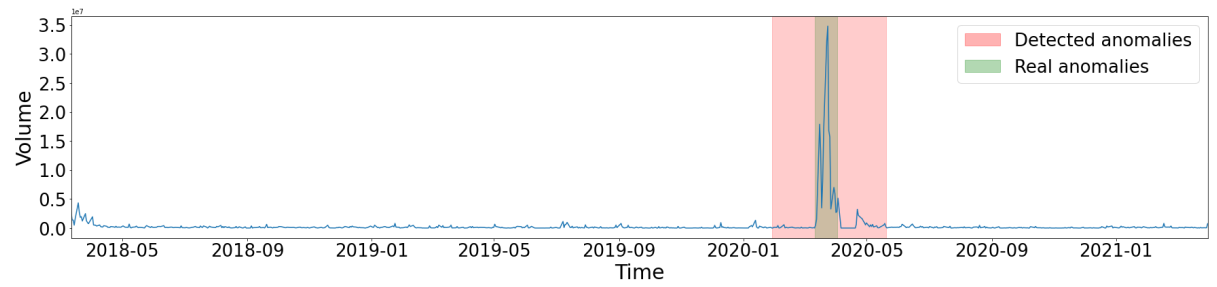

Traded volumes for GameStop (“GME”)

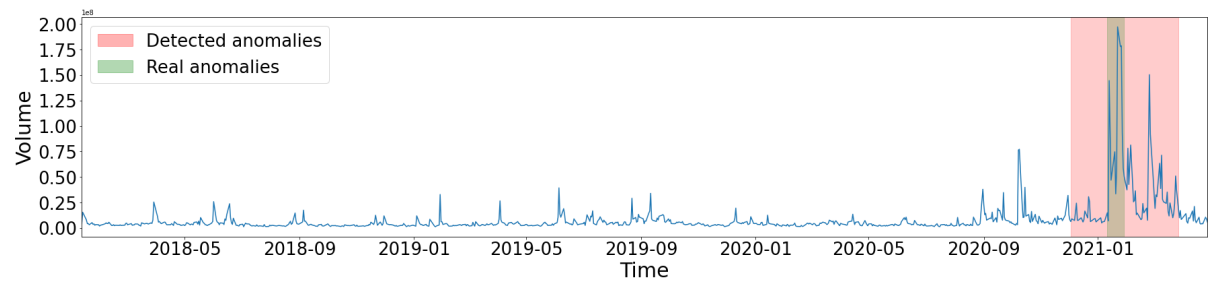

Fig. 2. Best Results for Each Data Set 


\section{Conclusion \& Future Work}

This paper has shared five large real-world, labelled data sets of anomalous stock market data. This is a valuable contribution to the field as real-world labelled data sets are rare, particularly for those showing allegedly illegal activities. New and emerging deep learning techniques have been applied to these data sets and compared with a statistical technique. This paper demonstrates that market manipulation is able to be detected using these techniques, with vary degrees of accuracy which a can be improved. The LSTM with Dynamic Thresholding appears to be particularly promising in this domain as it was able to identify contextual / local anomalies in the data and was able to detect anomalies quickly, taking seconds to score two years of trading data for each stock, which can often be a challenge with deep learning approaches. For future work on the issue of identifying market manipulation consideration should be given to applying hybrid methods combing both deep learning and statistical techniques, such as those described in Buda et al. [3], as ARIMA performed very well on point anomalies.

\section{References}

1. ASX Operations Pty Limited: Trading volumes. https://www2.asx.com.au/about/ market-statistics/trading-volumes\#monthly (Unknown date), (Accessed on 04/07/2021)

2. Australian Securities \& Investments Commission: Report REP 666 ASIC Enforcement Update: January to June 2020. https://www.asic.gov.au/media/5788492/ rep666-published-22-september-2020.pdf (September 2020), (Accessed on 04/07/2021)

3. Buda, T.S., Caglayan, B., Assem, H.: DeepAD: A Generic Framework Based on Deep Learning for Time Series Anomaly Detection. In: Phung, D., Tseng, V.S., Webb, G.I., Ho, B., Ganji, M., Rashidi, L. (eds.) Advances in Knowledge Discovery and Data Mining. pp. 577-588. Springer International Publishing (2018)

4. Fu, A.W.c., Leung, O.T.W., Keogh, E., Lin, J.: Finding time series discords based on haar transform. In: Li, X., Zaïane, O.R., Li, Z. (eds.) Advanced Data Mining and Applications. pp. 31-41. Springer Berlin Heidelberg, Berlin, Heidelberg (2006)

5. Geiger, A., Liu, D., Alnegheimish, S., Cuesta-Infante, A., Veeramachaneni, K.: TadGAN: Time Series Anomaly Detection Using Generative Adversarial Networks (2020)

6. Golmohammadi, K., Zaiane, O.R.: Time series contextual anomaly detection for detecting market manipulation in stock market. In: 2015 IEEE International Conference on Data Science and Advanced Analytics (DSAA). pp. 1-10 (2015)

7. Hundman, K., Constantinou, V., Laporte, C., Colwell, I., Soderstrom, T.: Detecting Spacecraft Anomalies Using LSTMs and Nonparametric Dynamic Thresholding. Proceedings of the 24th ACM SIGKDD International Conference on Knowledge Discovery \& Data Mining (Jul 2018)

8. Islam, S.R., Khaled Ghafoor, S., Eberle, W.: Mining Illegal Insider Trading of Stocks: A Proactive Approach. In: 2018 IEEE International Conference on Big Data (Big Data). pp. 1397-1406 (2018) 
9. Leangarun, T., Tangamchit, P., Thajchayapong, S.: Stock Price Manipulation Detection using Generative Adversarial Networks. In: 2018 IEEE Symposium Series on Computational Intelligence (SSCI). pp. 2104-2111 (2018)

10. Munir, M., Chattha, M.A., Dengel, A., Ahmed, S.: A Comparative Analysis of Traditional and Deep Learning-Based Anomaly Detection Methods for Streaming Data. In: 2019 18th IEEE International Conference On Machine Learning And Applications (ICMLA). pp. 561-566 (2019)

11. Munir, M., Siddiqui, S.A., Dengel, A., Ahmed, S.: DeepAnT: A Deep Learning Approach for Unsupervised Anomaly Detection in Time Series. IEEE Access 7, 1991-2005 (2019)

12. Pang, G., Shen, C., Cao, L., Hengel, A.V.D.: Deep Learning for Anomaly Detection. ACM Computing Surveys 54(2), 1-38 (Mar 2021)

13. PRNewswire: Aethlon Announces FDA Approval of IDE Supplement for COVID19 Patients. https://finance.yahoo.com/news/aethlon-announces-fda-approval-ide131900535.html (Jun 2020), (Accessed on 05/22/2021)

14. PRNewswire: Aethlon Medical Announces First Patient Treated in Firstin-Human Clinical Trial of HEMOPURIFIER R in Head and Neck Cancer. https://finance.yahoo.com/news/aethlon-medical-announces-first-patient130200071.html (Dec 2020), (Accessed on 05/22/2021)

15. Ramonas, A.: Covid-19 Scam Crackdown Prompts Spike in SEC Trading Suspensions. https://news.bloomberglaw.com/securities-law/covid-19-scamcrackdown-prompts-spike-in-sec-trading-suspensions (Jul 2020), (Accessed on $05 / 22 / 2021)$

16. Thorbecke, C.: GameStop timeline: A closer look at the saga that upended Wall Street. https://abcnews.go.com/Business/gamestop-timeline-closer-saga-upendedwall-street/story?id=75617315 (Feb 2021), (Accessed on 04/25/2021)

17. U.S Securities and Exchange Commission: SEC Charges Companies and CEO for Misleading COVID-19 Claims. https://www.sec.gov/news/press-release/2020-111 (May 2020), (Accessed on 04/25/2021)

18. U.S Securities and Exchange Commission: SEC Charges Companies for Misleading COVID-19 Claims. https://www.sec.gov/litigation/litreleases/2020/lr24819.htm (May 2020), (Accessed on 06/15/2021)

19. U.S Securities and Exchange Commission: Investor Alert 2021 - Look out for coronavirus-related investment scams. https://www.sec.gov/oiea/investor-alertsand-bulletins/ia_coronavirus (February 2021), (Accessed on 04/25/2021)

20. Wang, Q., Xu, W., Huang, X., Yang, K.: Enhancing intraday stock price manipulation detection by leveraging recurrent neural networks with ensemble learning. Neurocomputing 347, 46-58 (2019)

21. Wang, Z., Pi, D., Gao, Y.: A novel unsupervised time series discord detection algorithm in aircraft engine gearbox. In: Gan, G., Li, B., Li, X., Wang, S. (eds.) Advanced Data Mining and Applications. pp. 202-210. Springer International Publishing, Cham (2018)

22. Yu, P., Yan, X.: Stock price prediction based on deep neural networks. Neural Computing and Applications 32 (03 2020) 